\title{
Dual-passivation Strategy for Improved Ambient Stability of Perovskite Solar Cells
}

Ritesh Kant Gupta, ${ }^{a}$ Rabindranath Garai, ${ }^{b}$ Parameswar Krishnan Iyer*a,b

${ }^{a}$ Centre for Nanotechnology, Indian Institute of Technology Guwahati, Guwahati 781039, Assam, India

bDepartment of Chemistry, Indian Institute of Technology Guwahati, Guwahati 781039, Assam, India

Corresponding author Email: pki@iitg.ac.in 


\section{Experimental Section:}

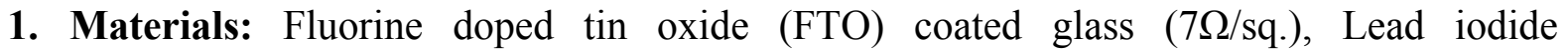
$\left(\mathrm{PbI}_{2}, 99 \%\right)$, p-toluene sulphonic acif (PTSA, >99\%), Polystyrene (PS, >99\%), anhydrous DMF, anhydrous DMSO, Rhodamine-101 and silver was purchased from Sigma Aldrich. Methylammonium iodide (MAI, 99\%) was purchased from Greatcell Solar. (6,6)-PhenylC61-butyric Acid Methyl Ester (PCBM, >99.5\%) was purchased from LUMTEC for electron transport layer. Poly(3,4-ethylenedioxythiophene)-poly(styrenesulfonate) (PEDOT:PSS, PVP AI 4083) was procured from Clevios for hole-transporting layer. All the materials were used as received until otherwise specified.

2. Device Fabrication: The solar cell devices were fabricated on FTO coated glass which was pre-patterned and cleaned sequentially in soap solution, de-ionized water, acetone and isopropanol. The substrate was then dried under $\mathrm{N}_{2}$ gas and UV-ozone treated before further processing. NiOx precursor solution for hole-transporting layer was prepared by dissolving $1 \mathrm{M}$ Nickel nitrate hexahydrate and $1 \mathrm{M}$ Ethylenediamine in $1 \mathrm{~mL}$ Ethylene Glycol. Then the NiOx layer was coated as hole transporting layer on the cleaned FTO. $\mathrm{NiOx}$ precursor solution was spin coated onto the FTO substrates at $3000 \mathrm{rpm}$ for $40 \mathrm{sec}$. Afterwards the substrates were post-annealed at $300{ }^{\circ} \mathrm{C}$ for $60 \mathrm{~min}$ in ambient air. The $\mathrm{MAPbI}_{3}$ precursor solution was prepared in a glovebox by dissolving MAI and PbI2 was mixed in the ratio 1:1 (Molar concentration 1.26) in $\gamma$-Butyrolactone and DMSO (7:3, $\mathrm{v} / \mathrm{v}$ ) The solution was heated overnight prior to spin coating. For the modified device, different concentrations of PTSA additive were added to the precursor solution [1 $\mathrm{mg} / \mathrm{mL}, 2 \mathrm{mg} / \mathrm{mL}$ and $3 \mathrm{mg} / \mathrm{mL}$ ]. PS was coated on top of perovskite layer with either 2 $\mathrm{mg} / \mathrm{mL}$ or $4 \mathrm{mg} / \mathrm{mL}$ concentration in chlorobenzene The precursor solution was spin coated on the NiOx coated FTO in a two-step spin coating process i.e. $750 \mathrm{rpm}$ for $20 \mathrm{sec}$ and $4000 \mathrm{rpm}$ for $60 \mathrm{sec}$. In the second step $160 \mathrm{~mL}$ anhydrous Toluene was dripped after $20 \mathrm{sec}$ as antisolvent and after that the substrates were annealed at $80^{\circ} \mathrm{C}$ for $10 \mathrm{~min} .12$ $\mathrm{mg} / \mathrm{mL}$ PCBM in chlorobenzene was used as electron transport layer which was spincoated at $1200 \mathrm{rpm}$ for $30 \mathrm{sec}$ on top of perovskite. Lastly, thin layer of rhodamine-101 in isopropanol was spin-coated at $4000 \mathrm{rpm}$ for $30 \mathrm{sec} .100 \mathrm{~nm}$ of $\mathrm{Ag}$ was used at cathode metal was vacuum deposited at $10^{-6}$ mbar pressure. The overall device architecture was FTO/NiOx/Perovskite/PCBM/Rhodamine-101/Ag.

3. Characterization: The perovskite films were characterized by X-ray diffraction (XRD, Rigaku Micromax-007HF diffractometer equipped with $\mathrm{Cu} \mathrm{K} \alpha 1$ irradiation $[\lambda=1.54184$ $\AA]$ ), UV-vis absorption spectroscopy (PerkinElmer Lamda-35), field emission scanning electron microscopy (FESEM, JEOL JSM-7610F), and. The current density-voltage $(\mathrm{J}-\mathrm{V})$ characteristic curves were recorded with a Keithley 2400 source meter in argon atmosphere for dark characterization. All light characterization was done by illuminating the device with a solar simulator (Newport, Oriel Sol 3A solar simulator, AM 1.5G, $100 \mathrm{~mW} \mathrm{~cm}-2)$ using a shadow mask. The steady state current analysis, Mott-Scottky and impedance spectroscopic measurement were performed using an electrochemical 
workstation (CH Instruments 760D). An Oriel IQE-200 instrument was used to record the incident photon-to-current efficiency (IPCE) in ambient condition.

4. Equations used: Hysteresis index (HI) was calculated as per the following equation:

$$
H I=\frac{P C E_{\text {forward }}-P C E_{\text {reverse }}}{P C E_{\text {forward }}} X 100
$$

From the obtained $\mathrm{V}_{\text {TFL }}$ values, the trap density $\left(\mathrm{N}_{\text {trap }}\right)$ is calculated using the equation:

$$
N_{\text {trap }}=\frac{2 \varepsilon \varepsilon_{0} V_{T F L}}{e L^{2}}
$$

where, $\varepsilon$ is the dielectric constant of perovskite, $\varepsilon_{0}$ is permittivity of free space, $\mathrm{e}$ is the charge of electron and $\mathrm{L}$ is the thickness.

Urbach energy $(\mathrm{Eu})$ is represented by the equation:

$$
\alpha=\alpha_{0} \exp \left(\frac{E}{E_{u}}\right)
$$

where, $\alpha$ is the absorption coefficient, $\alpha_{0}$ is a constant and $\mathrm{E}$ is the photon energy. Therefore, the inverse slope in the plot of $\ln (\alpha)$ versus $E$ can project the value of $E_{u}$.
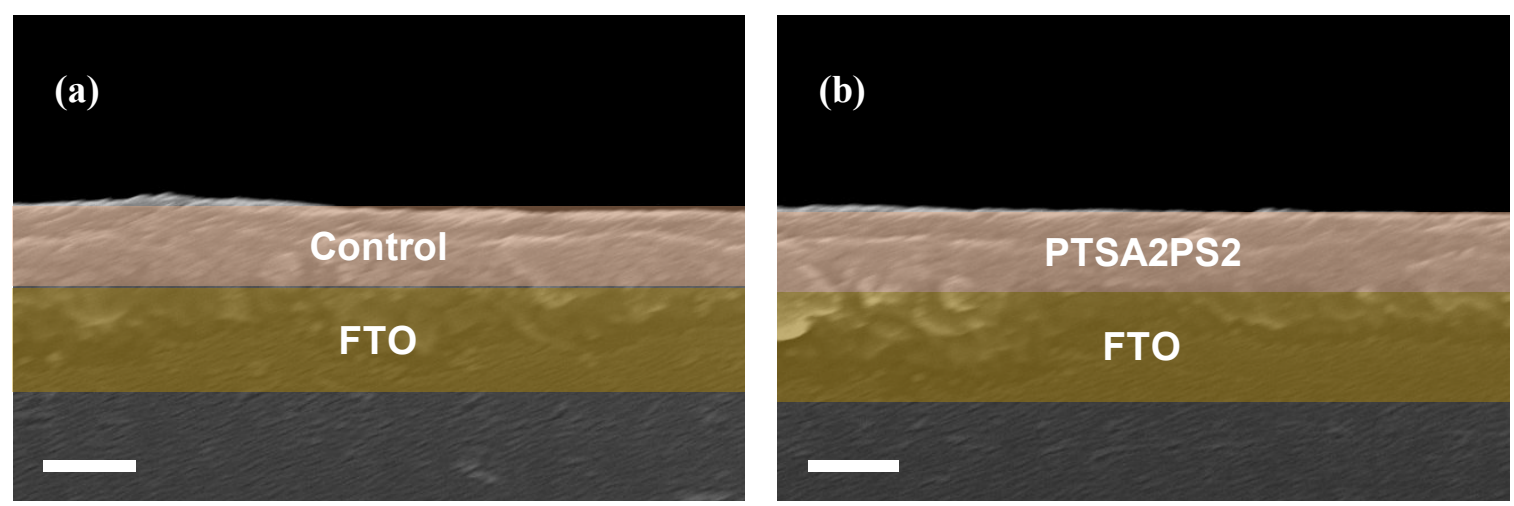

Figure S1. Cross-sectional FESEM of (a) control, and (b) PTSA2PS2 perovskite films (scale bar $=500 \mathrm{~nm})$ 


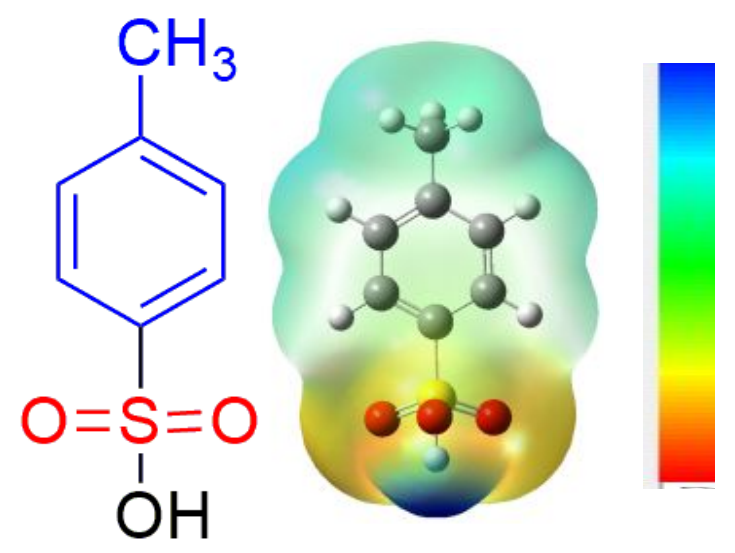

Figure S2. Electro-static profile of PTSA.

Table S1. Photovoltaic parameters of all types of fabricated using dual-passivation approach

\begin{tabular}{|l|l|l|l|c|}
\hline \multicolumn{1}{|c|}{ Device Type } & Jsc, $^{-2} \mathrm{~m} \mathrm{~cm}^{-2}$ & Voc, $\mathrm{V}$ & $\mathrm{FF}, \%$ & PCE, $\%$ \\
\hline Control & 21.25 & 1.008 & 70.7 & $15.14(13.65 \pm 0.73)$ \\
\hline PTSA2 & 23.39 & 1.071 & 73.9 & $18.51(17.36 \pm 0.69)$ \\
\hline PS2 & 22.88 & 1.053 & 76.0 & $18.31(17.11 \pm 0.68)$ \\
\hline PTSA1PS2 & 22.17 & 1.057 & 74.3 & $17.47(16.15 \pm 0.63)$ \\
\hline PTSA1PS4 & 21.67 & 1.041 & 73.2 & $16.51(15.44 \pm 0.60)$ \\
\hline PTSA2PS2 & 23.25 & 1.114 & 79.6 & $20.62(19.90 \pm 0.34)$ \\
\hline PTSA2PS4 & 22.58 & 1.094 & 77.8 & $19.21(18.29 \pm 0.49)$ \\
\hline PTSA3PS2 & 21.92 & 1.124 & 76.1 & $18.75(17.60 \pm 0.54)$ \\
\hline PTSA3PS4 & 20.92 & 1.107 & 74.3 & $17.21(16.05 \pm 0.51)$ \\
\hline
\end{tabular}
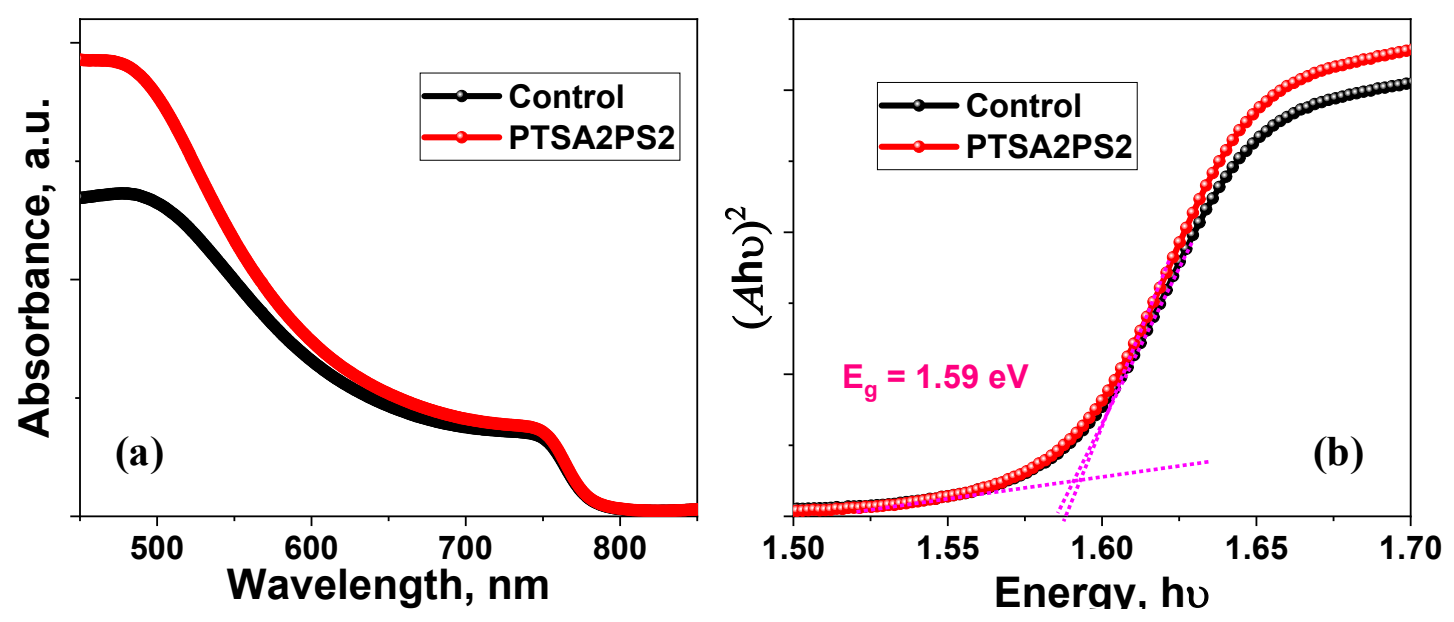

Figure S3. (a) UV-vis absorbance, and (b) TauC plot. 


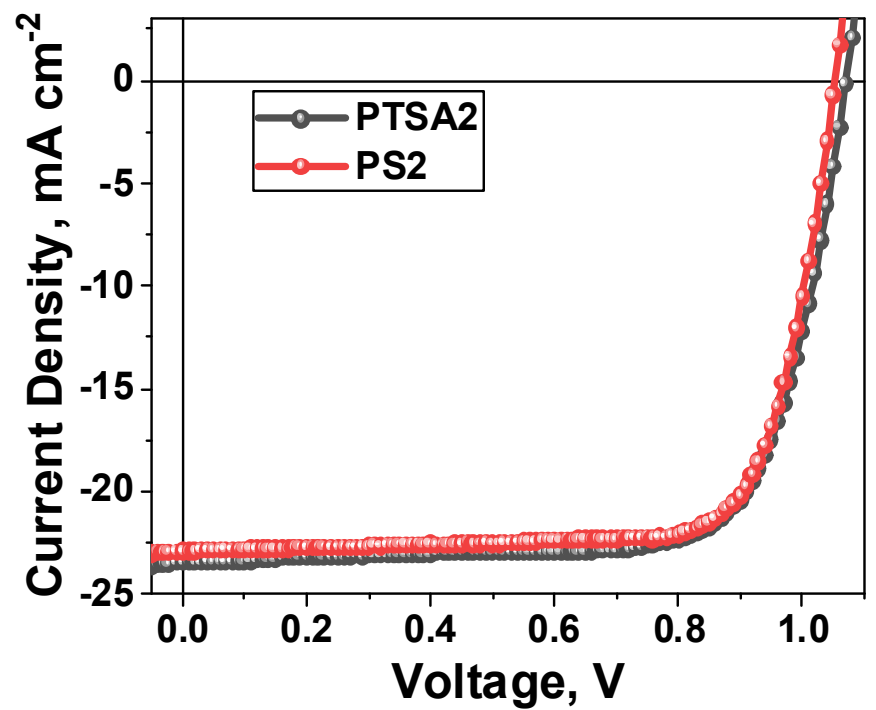

Figure S4. JV characteristics curve of PTSA2 and PS2.

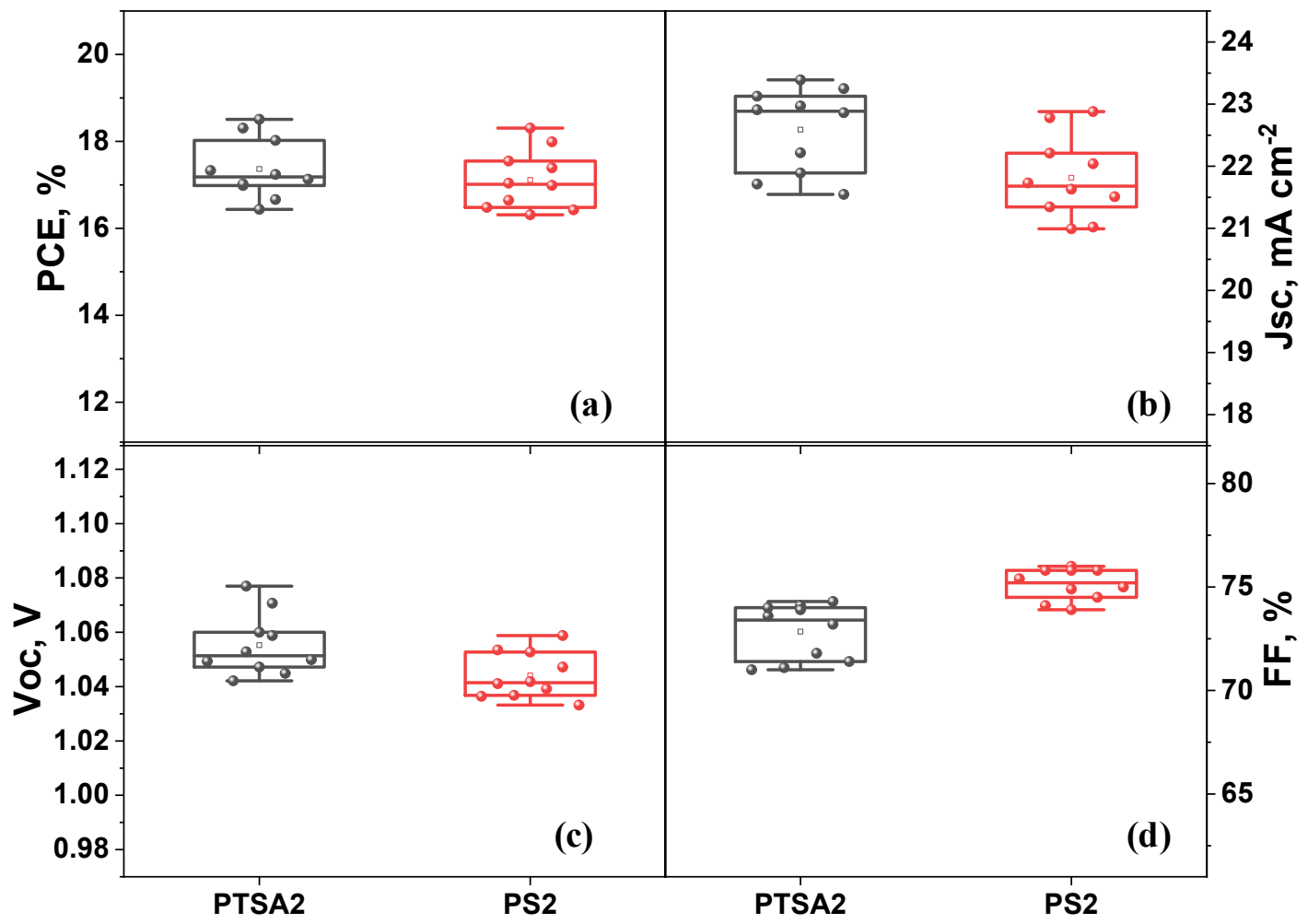

Figure S5. Box chart showing variation in (a) PCE, (b) Jsc, (c) Voc, and (d) FF for 10 devices of PTSA2 and PS2. 


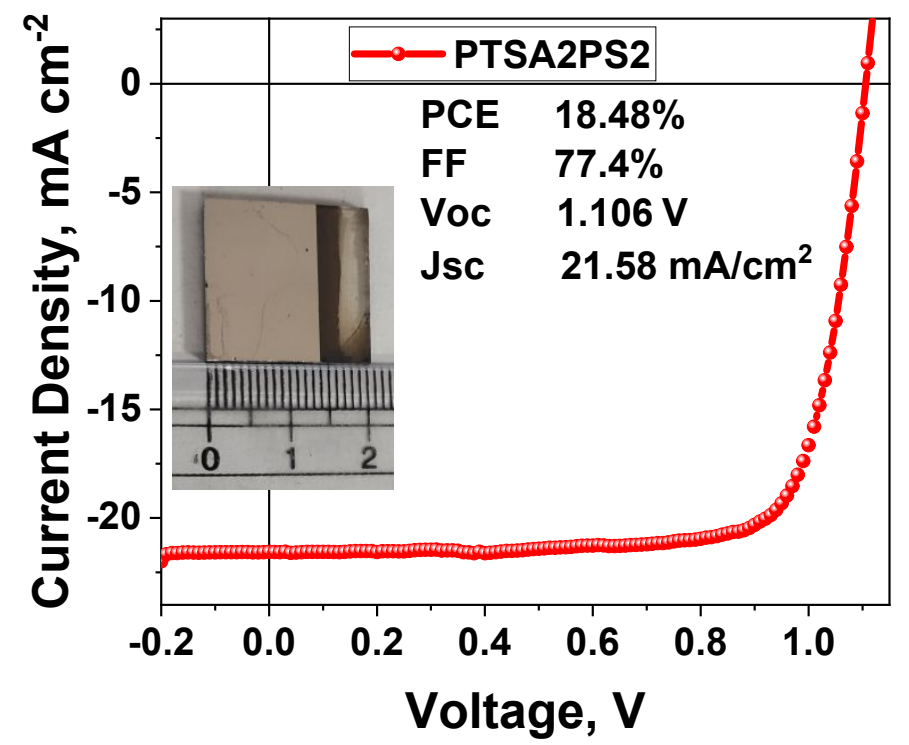

Figure S6. JV curve of PTSA2PS2 based large-area device.

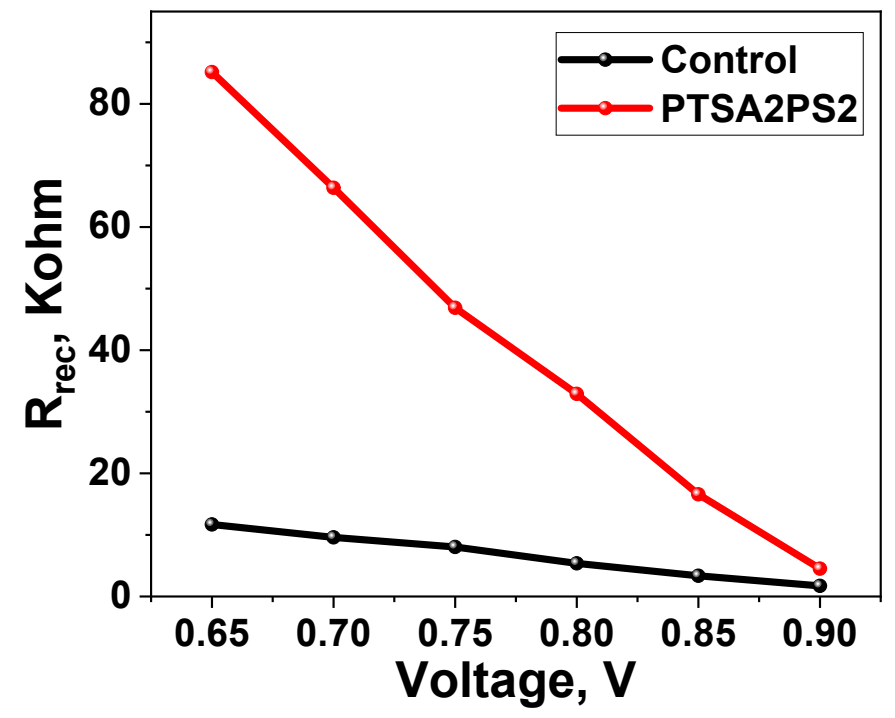

Figure S7. Resistance versus voltage plot. 


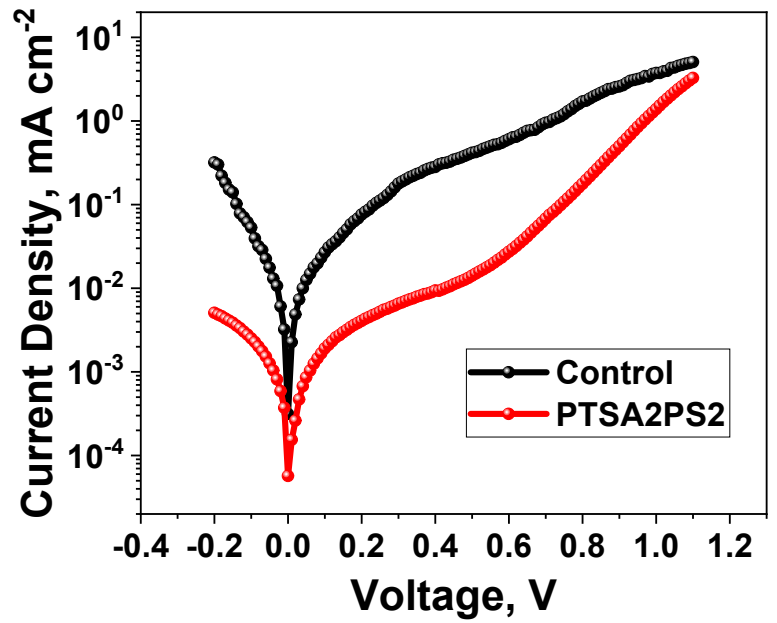

Figure S8. Dark JV plots of the solar cell devices.

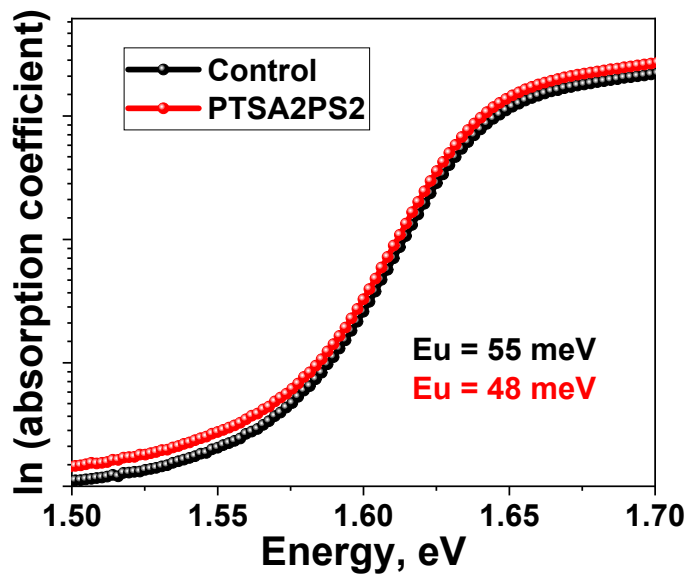

Figure S9. $\ln$ (absorption coefficient) versus energy plot to calculate Urbach energy.

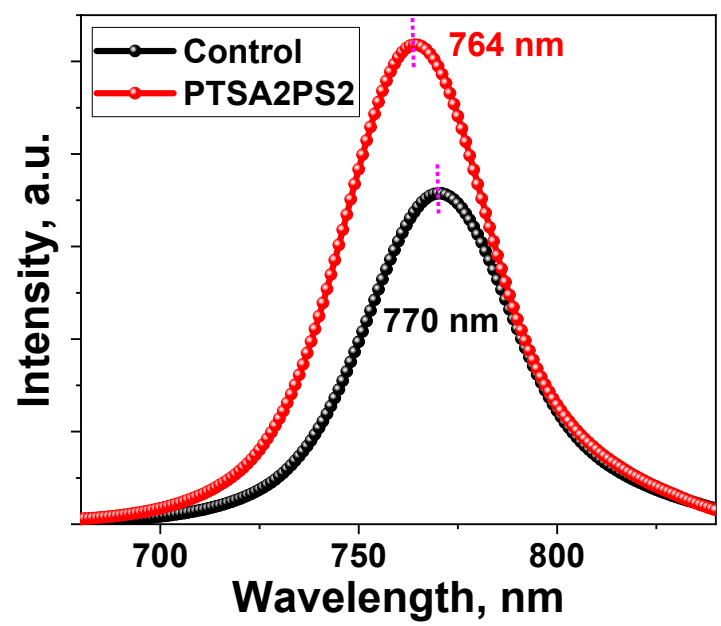

Figure S10. PL spectra of the perovskite films. 


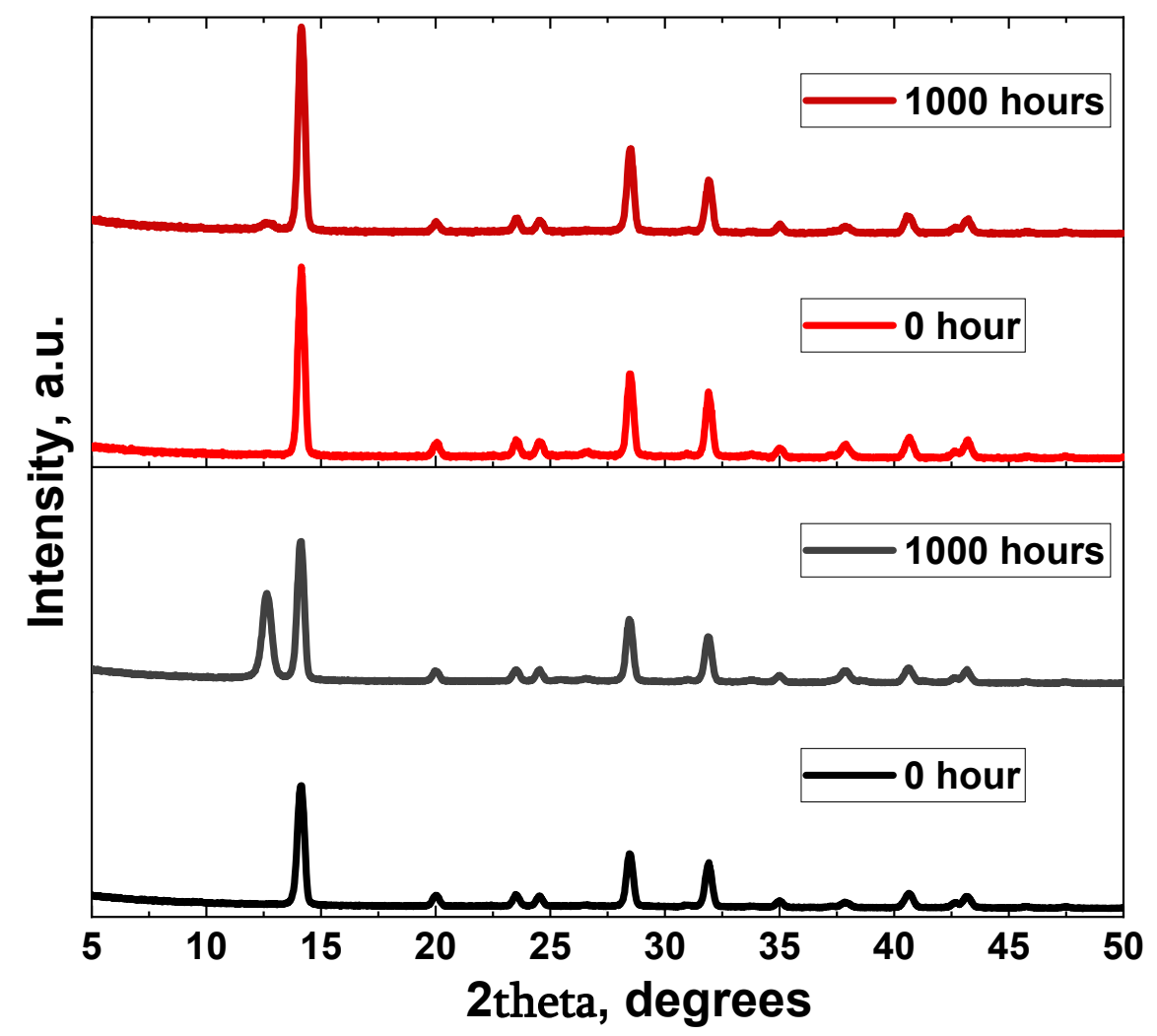

Figure S11. XRD patterns of the perovskite films to check ambient stability

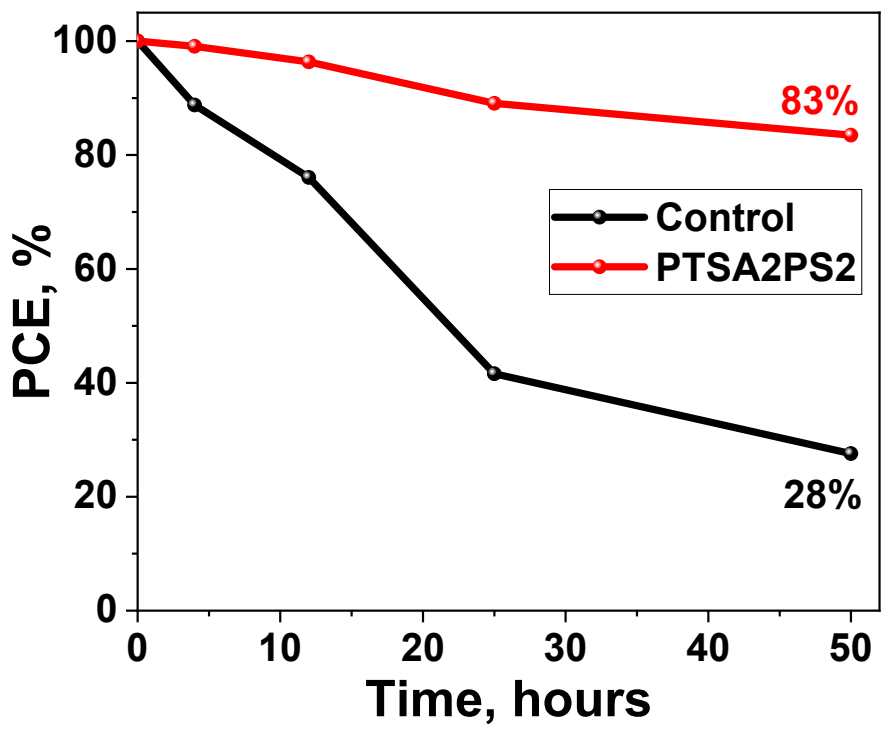

Figure S12. Thermal stability of the devices showing normalized PCE. 
Table S2 Recent literature with $\mathrm{NiO}_{\mathrm{x}}$ as hole transport layer, $\mathrm{MAPbI}_{3}$ perovskite active layer and PCBM as electron transport layer.

\begin{tabular}{|c|c|c|c|c|}
\hline $\begin{array}{l}\text { Sl. } \\
\text { No. }\end{array}$ & Device configuration & $\begin{array}{l}\text { PCE (Large-area } \\
\text { PCE) [\%] }\end{array}$ & Ambient Stability & Ref \\
\hline 1. & FTO/NiOx/CH $3 \mathrm{NH}_{3} \mathrm{PbI}_{3} / \mathrm{PCBM} /$ Rhodamine-101/Ag & $\begin{array}{c}20.62 \\
(18.43)\end{array}$ & $\begin{array}{c}\text { 93\% stable after } 1000 \text { hours (R.H. } \\
\mathbf{5 0} \pm \mathbf{5 \%} \text { ) }\end{array}$ & $\begin{array}{l}\text { This } \\
\text { work }\end{array}$ \\
\hline 2. & FTO/ $\mathrm{NiO}_{\mathrm{x}} / \mathrm{CH}_{3} \mathrm{NH}_{3} \mathrm{PbI}_{3} / \mathrm{PCBM} / \mathrm{PEI} / \mathrm{Ag}$ & $20.61(\mathrm{NA})$ & $90 \%$ stable after 250 hours (R.H. $30 \%$ ) & S1 \\
\hline 3. & $\mathrm{FTO} / \mathrm{NiO}_{\mathrm{x}} / \mathrm{CH}_{3} \mathrm{NH}_{3} \mathrm{PbI}_{3} / \mathrm{PCBM} / \mathrm{BCP} / \mathrm{Ag}$ & 20.43 (NA) & $86 \%$ stable after 30 days (R.H. $30 \%$ ) & S2 \\
\hline 4. & $\mathrm{ITO} / \mathrm{NiO}_{\mathrm{x}} / \mathrm{CH}_{3} \mathrm{NH}_{3} \mathrm{PbI}_{3-\mathrm{x}} \mathrm{Cl}_{\mathrm{x}} / \mathrm{C} 60 / \mathrm{BCP} / \mathrm{Ag}$ & $20.10(\mathrm{NA})$ & (NA) & S3 \\
\hline 5. & $\mathrm{ITO} / \mathrm{NiO}_{\mathrm{x}} / \mathrm{CH}_{3} \mathrm{NH}_{3} \mathrm{PbI}_{3} / \mathrm{PCBM} / \mathrm{PEI} / \mathrm{Ag}$ & $20.05(\mathrm{NA})$ & $80 \%$ stable after 1000 hours (R.H. $40 \%$ ) & S4 \\
\hline 6. & ITO/ $\mathrm{NiO}_{\mathrm{x}} / \mathrm{CH}_{3} \mathrm{NH}_{3} \mathrm{PbI}_{3} / \mathrm{PCBM} / \mathrm{BCP} / \mathrm{Ag}$ & 19.80 (NA) & $90 \%$ stable after 30 days (R.H. $45 \%$ ) & S5 \\
\hline 7. & $\mathrm{ITO} / \mathrm{NiO}_{\mathrm{x}} / \mathrm{CH}_{3} \mathrm{NH}_{3} \mathrm{PbI}_{3} / \mathrm{PCBM} / \mathrm{BCP} / \mathrm{Ag}$ & $19.50(\mathrm{NA})$ & Highly Stable over 500 hours (R.H. $40 \%$ ). & S6 \\
\hline 8. & $\mathrm{ITO} / \mathrm{NiO}_{\mathrm{x}} / \mathrm{CH}_{3} \mathrm{NH}_{3} \mathrm{PbI}_{3-\mathrm{x}} \mathrm{Cl}_{\mathrm{x}} / \mathrm{PCBM} / \mathrm{BCP} / \mathrm{Ag}$ & $19.34(\mathrm{NA})$ & $85 \%$ stable after 50 days (R.H. $40 \%$ ) & S7 \\
\hline 9. & FTO/ $\mathrm{NiO}_{x} / \mathrm{CH}_{3} \mathrm{NH}_{3} \mathrm{PbI}_{3} / \mathrm{PCBM} /$ Rhodamine101/Ag & $19.06(\mathrm{NA})$ & $80 \%$ stable after 1000 hours (R.H. $30-40 \%$ ) & S8 \\
\hline 10. & $\mathrm{ITO} / \mathrm{NiO}_{\mathrm{x}} / \mathrm{CH}_{3} \mathrm{NH}_{3} \mathrm{PbI}_{3} / \mathrm{PC} 61 \mathrm{BM} / \mathrm{BCP} / \mathrm{Ag}$ & $18.96(\mathrm{NA})$ & $84 \%$ stable after 216 hours (R.H. $30 \%$ ) & S9 \\
\hline 11. & $\mathrm{FTO} / \mathrm{NiO}_{\mathrm{x}} / \mathrm{CH}_{3} \mathrm{NH}_{3} \mathrm{PbI}_{3} / \mathrm{PCBM} / \mathrm{PEI} / \mathrm{Ag}$ & $18.84(\mathrm{NA})$ & $80 \%$ stable after 400 hours (glove box) & $\mathrm{S} 10$ \\
\hline 12. & $\mathrm{FTO} / \mathrm{Li}-\mathrm{NiO}_{\mathrm{x}} / \mathrm{CH}_{3} \mathrm{NH}_{3} \mathrm{PbI}_{3} / \mathrm{PCBM} / \mathrm{BCP} / \mathrm{Ag}$ & $18.35(\mathrm{NA})$ & $80 \%$ stable after 35 days (R.H. $10 \pm 5 \%$ ) & S11 \\
\hline 13. & $\mathrm{FTO} / \mathrm{NiO}_{\mathrm{x}} / \mathrm{CH}_{3} \mathrm{NH}_{3} \mathrm{PbI}_{3} / \mathrm{PCBM} / \mathrm{Ag}$ & $\begin{array}{c}18.30 \\
\mathbf{( 1 6 . 2 0 )}\end{array}$ & $95 \%$ stable after 7 days (R.H. $20 \%$ ) & $\mathrm{S} 12$ \\
\hline 14. & ITO/NiOx/ $\mathrm{CH}_{3} \mathrm{NH}_{3} \mathrm{PbI}_{3} / \mathrm{PCBM} / \mathrm{BCP} / \mathrm{Ag}$ & $18.24(\mathrm{NA})$ & $74 \%$ stable after 48 hours (R.H. $80 \%$ ) & $\mathrm{S} 13$ \\
\hline 15. & ITO/ $\mathrm{NiO}_{\mathrm{x}} / \mathrm{CH}_{3} \mathrm{NH}_{3} \mathrm{PbI}_{3} / \mathrm{PCBM} / \mathrm{Ca}(\mathrm{acac})_{2} / \mathrm{Ag}$ & $18.23(\mathrm{NA})$ & $85 \%$ stable after 30 days (glove box) & S14 \\
\hline 16. & $\mathrm{FTO} / \mathrm{NiO}_{\mathrm{x}} / \mathrm{CH}_{3} \mathrm{NH}_{3} \mathrm{PbI}_{3-\mathrm{x}} \mathrm{Cl}_{\mathrm{x}} / \mathrm{PCBM} / \mathrm{PEI} / \mathrm{Ag}$ & $\begin{array}{c}18.2 \\
\mathbf{( 1 5 . 4 0 )}\end{array}$ & (NA) & $\mathrm{S} 15$ \\
\hline 17. & ITO/NiOx $/ \mathrm{CH}_{3} \mathrm{NH}_{3} \mathrm{PbI}_{3} / \mathrm{PCBM} / \mathrm{Ag}$ & $\begin{array}{c}18.00 \\
\mathbf{( 1 6 . 3 9 )}\end{array}$ & $86 \%$ stable after 480 hours (R.H. $30 \%$ ) & S16 \\
\hline 18. & $\mathrm{FTO} / \mathrm{NiO}_{\mathrm{x}} / \mathrm{CH}_{3} \mathrm{NH}_{3} \mathrm{PbI}_{3} / \mathrm{PCBM} / \mathrm{PEI} / \mathrm{Ag}$ & $17.98(\mathrm{NA})$ & $\begin{array}{c}70 \% \text { stable after } 60 \mathrm{~min} \text { (R.H. } 50-60 \% \\
\text { under light) }\end{array}$ & S17 \\
\hline 19. & $\mathrm{ITO} / \mathrm{NiO}_{\mathrm{x}} / \mathrm{CH}_{3} \mathrm{NH}_{3} \mathrm{PbI}_{3} / \mathrm{PCBM} / \mathrm{BCP} / \mathrm{Ag}$ & $17.85(\mathrm{NA})$ & Stable for 500 hours in glove box. & S18 \\
\hline 20. & ITO/ $\mathrm{NiO}_{\mathrm{x}} / \mathrm{CH}_{3} \mathrm{NH}_{3} \mathrm{PbI}_{3} / \mathrm{PCBM} / \mathrm{PTN}-\mathrm{Br} / \mathrm{Ag}$ & 17.44 (NA) & $88 \%$ stable after 20 days (glove box) & S19 \\
\hline 21. & $\mathrm{FTO} / \mathrm{NiO}_{\mathrm{x}} / \mathrm{CH}_{3} \mathrm{NH}_{3} \mathrm{PbI}_{3} / \mathrm{PCBM} / \mathrm{PEI} / \mathrm{Ag}$ & $17.24(\mathrm{NA})$ & (NA) & S20 \\
\hline 22. & ITO/ $\mathrm{NiO}_{\mathrm{x}} / \mathrm{CH}_{3} \mathrm{NH}_{3} \mathrm{PbI}_{3} / \mathrm{PCBM} / \mathrm{BCP} / \mathrm{Ag}$ & $16.6(\mathrm{NA})$ & $78 \%$ stable after 35 days(R.H. $30-40 \%$ ) & S21 \\
\hline
\end{tabular}

NA- Not Available

\section{References:}

[S1] Y. Cui, S. Wang, C. Li, A. Wang, J. Ren, C. Yang, B. Chen, Z. Wang, F. Hao, Green Chem., 2021, 23, 3633. 
[S2] T. Wu, Y. Wang, X. Li, Y. Wu, X. Meng, D. Cui, X. Yang, L. Han, Adv. Energy Mater.2019, 9, 1803766.

[S3] J. Cheng, H. Zhang, S. Zhang, D. Ouyang, Z. Huang, M. K. Nazeeruddin, J. Hou, W. C. H. Choy, J. Mater. Chem. A, 2018, 6, 23865.

[S4] C. Li, J. Hu, S. Wang, J. Ren, B. Chen, T. Pan, X. Niu, F. Hao, J. Phys. Chem. Lett.2021, $12,4569$.

[S5] H.-C. Chen, J.-M. Lan, H.-L. Hsu, C.-W. Li, T.-S. Shieh, K.-T. Wong, C.-P. Chen, Mater. Chem. Front., 2021, 5, 3378.

[S6] H.-L. Hsu, H.-T. Hsiao, T.-Y. Juang, B.-H. Jiang, S.-C. Chen, R.-J. Jeng, C.-P. Chen, Adv. Energy Mater.2018, 8, 1802323.

[S7] F. Zhang, S. Guang, M. Ye, K. Xu, W. Guo, X. Liu, H. Xu, ACS Appl. Mater. Interfaces, 2018, 10, 36841.

[S8] R. Garai, M. A. Afroz, R. K. Gupta, P. K. Iyer, Adv. Sustain. Syst., 2020, 4, 2000078.

[S9] S.-Y. Wang, C.-P. Chen, C.-L. Chung, C.-W. Hsu, H.-L. Hsu, T.-H. Wu, J.-Y. Zhuang, C.-J. Chang, H. M. Chen, Y. J. Chang, ACS Appl. Mater. Interfaces, 2019, 11, 40050.

[S10] K.-C. Hsiao, M.-H. Jao, B.-T. Li, T.-H. Lin, S. H.-C. Liao, M.-C. Wu, W.-F. Su, ACS Appl. Energy Mater., 2019, 2, 4821.

[S11] Y. Guo, J. Ma, H. Lei, F. Yao, B. Li, L. Xiong, G. Fang, J. Mater. Chem. A, 2018, 6, 5919.

[S12] W. Chen, Y. Wu, Y. Yue, J. Liu, W. Zhang, X. Yang, H. Chen, E. Bi, I. Ashraful, M. Grätzel, L. Han, Science, 2015, DOI: 10.1126/science.aad1015aad1015.

[S13] Y. Ma, H. Zhang, Y. Zhang, R. Hu, M. Jiang, R. Zhang, H. Lv, J. Tian, L. Chu, J. Zhang, Q. Xue, H.-L. Yip, R. Xia, X. a. Li, W. Huang, ACS Appl. Mater. Interfaces, 2019, 11, 3044.

[S14] Z. Q. Zhao, S. You, J. Huang, L. Yuan, Z. Y. Xiao, Y. Cao, N. Cheng, L. Hu, J. F. Liu, B. H. Yu, J. Mater. Chem. C, 2019, 7, 9735.

[S15] H.-C. Liao, P. Guo, C.-P. Hsu, M. Lin, B. Wang, L. Zeng, W. Huang, C. M. M. Soe, W.F. Su, M. J. Bedzyk, M. R. Wasielewski, A. Facchetti, R. P. H. Chang, M. G. Kanatzidis, T. J. Marks, Adv. Energy Mater., 2017, 7, 1601660.

[S16] C. Li, A. Wang, L. Xie, X. Deng, K. Liao, J.-a. Yang, Y. Xiang, F. Hao, J. Mater. Chem. C, 2020, 8, 3217.

[S17] H. Zhang, H. Chen, C. C. Stoumpos, J. Ren, Q. Hou, X. Li, J. Li, H. He, H. Lin, J. Wang, F. Hao, M. G. Kanatzidis, ACS Appl. Mater. Interfaces, 2018, 10, 42436.

[S18] Y.-Y. Yu, C. Tseng, W.-C. Chien, H.-L. Hsu, C.-P. Chen, J. Phys. Chem. C, 2019, 123, 23826. 
[S19] J. Tu, C. Liu, Y. Fan, F. Liu, K. Chang, Z. Xu, Q. Li, Y. Chen, Z. Li, J. Mater. Chem. A, 2019, 7, 15662.

[S20] K. Liao, J.-a. Yang, C. Li, T. Li, F. Hao, ACS Appl. Mater. Interfaces, 2019, 11, 39882.

[S21] C. Liu, Z. Huang, X. Hu, X. Meng, L. Huang, J. Xiong, L. Tan, Y. Chen, ACS Appl. Mater. Interfaces, 2018, 10, 1909. 